\title{
Very low bilirubin after portoenterostomy improves survival of the native liver in patients with biliary atresia by deferring liver fibrogenesis
}

\author{
Maria Hukkinen, MD, PhD ${ }^{a}$, Anna Kerola, MDa, Jouko Lohi, MD, PhD ${ }^{b}$, \\ Timo Jahnukainen, $\mathrm{MD}, \mathrm{PhD}^{\mathrm{c}}$, Päivi Heikkilä, $\mathrm{MD}, \mathrm{PhD}^{\mathrm{b}}$, Mikko P. Pakarinen, $\mathrm{MD}, \mathrm{PhD}^{\mathrm{a}, *}$ \\ a Pediatric Liver and Gut Research Group and Section of Pediatric Surgery, Children's Hospital, Helsinki University Hospital, Finland \\ ${ }^{\mathrm{b}}$ Department of Pathology, HUSLAB, Helsinki University Hospital, Finland \\ ${ }^{\mathrm{C}}$ Department of Pediatric Nephrology and Transplantation, Children's Hospital, Helsinki University Hospital, Finland
}

\section{A R T I C L E I N F O}

Article history:

Accepted 23 October 2018

Available online $\mathrm{xxx}$

\begin{abstract}
A B S T R A C T
Background: Progression of fibrosis and ensuing complications determine the postoperative course of patients operated on for biliary atresia. We evaluated predictors of the progression of fibrosis in the native liver after operative treatment.

Methods: Among patients whose bilirubin decreased to $<34 \mu \mathrm{mol} / \mathrm{L}$ after portoenterostomy $(n=41)$, predictors of follow-up cirrhosis and the progression of fibrosis were analyzed with logistic regression and survival of their native liver was evaluated with the Kaplan-Meier method. Areas under receiving operating characteristic were used to define optimal cutoffs.

Results: After median follow-up of 5.2 years (interquartile range 1.6-10.2) after portoenterostomy, liver biopsies showed cirrhosis in $53 \%$ of patients, and the Metavir stage remained stable or decreased in $38 \%$. The development of cirrhosis was predicted by total or conjugated bilirubin $\geq 170 / 120 \mu \mathrm{mol} / \mathrm{L}$ at the time of portoenterostomy $(P \leq .009)$; normalization of bilirubin within 1.9 months $(P=.002)$; total or conjugated bilirubin $\geq 12.5 / 7.5 \mu \mathrm{mol} / \mathrm{L}(P=.002)$ and aspartate aminotransferase-to-platelet ratio $\geq 0.55$ at 3 months postoperatively $(P=.001)$; and total or conjugated bilirubin $\geq 7.5 / 2.5 \mu \mathrm{mol} / \mathrm{L}(P \leq .001)$, aspartate aminotransferase-to-platelet ratio $\geq 0.63(P=.004)$, and gamma glutamyl transferase $\geq 266 \mathrm{U} / \mathrm{L}$ $(P=.007)$ at 6 months postoperatively. In multiple regression analysis, conjugated bilirubin $\geq 2.5 \mu \mathrm{mol} / \mathrm{L}$ at 6 months increased the risk of cirrhosis 35-fold $(P=.020)$, and other predictors were not predictive. Total or conjugated bilirubin $<12.5 / 7.5 \mu \mathrm{mol} / \mathrm{L}(P \leq .014)$, aspartate aminotransferase-to-platelet ratio < 0.55 at 3 months $(P=.006)$, and total or conjugated bilirubin $<7.5 / 2.5 \mu \mathrm{mol} / \mathrm{L}$ at 6 months postoperatively $(P \leq .014)$ were the strongest predictors of a stable, nonprogressive fibrosis. Decreases in total or conjugated bilirubin to $<12.5 / 7.5 \mu \mathrm{mol} / \mathrm{L}$ by 3 months and to $<7.5 / 2.5 \mu \mathrm{mol} / \mathrm{L}$ by 6 months improved survival of the native liver ( $\log$-rank $P \leq .025)$. Age at follow-up or at portoenterostomy, anatomic type of biliary atresia, use of postoperative steroids, and episodes of cholangitis were unrelated to the progression of fibrosis or the development of cirrhosis ( $P=$ not significant).

Conclusion: Among patients whose serum bilirubin normalizes after portoenterostomy, its rapid decrease to very low levels prolongs the survival of their native liver by delaying the progression of fibrosis.
\end{abstract}

(c) 2018 Elsevier Inc. All rights reserved.

\section{Introduction}

Biliary atresia (BA) is a rare cholangiopathy of the newborn, characterized by fibroinflammatory obliteration of the biliary tree,

\footnotetext{
th Supported with research grants by the Sigrid Jusélius Foundation, the Finnish Pediatric Research Foundation, and the Helsinki University Central Hospital Grant.

* Corresponding author: Helsinki University Hospital, Children's Hospital, P.O. Box 281, 00029 HUS, Helsinki, Finland.

E-mail address: mikko.pakarinen@hus.fi (M.P. Pakarinen).
}

which results in cholestasis and rapidly progressive biliary cirrhosis. After portoenterostomy (PEostomy) aiming to restore bile flow, more than half of patients achieve clearance of jaundice (COJ), effectively postponing the need for liver transplantation (LT).${ }^{1-4}$ Despite normalization of serum bilirubin by successful PEostomy, however, liver injury progresses chronically, resulting almost invariably in cirrhosis ${ }^{5-8}$ and warranting LT in $75 \%-80 \%$ of patients by adult age. $6,8,9$

As in other chronic liver diseases, the postoperative course of BA is primarily determined by progression of liver fibrosis and 
ensuing complications. ${ }^{10-12}$ Patients who fail to clear their jaundice after PEostomy develop cirrhosis and end-stage liver disease rapidly. The disease course is far more variable after a successful PEostomy, which enables prolonged survival with the native liver. As long-term fibrosis of the native liver has rarely been studied, no reliable means exist to predict in which patients liver fibrosis progresses, remains stable, or even resolves after clearance of jaundice (COJ), although noninvasive methods may be useful in estimating the current stage of fibrosis in the liver. ${ }^{12,13}$ Although overall survival of the native liver after PEostomy depends strongly on the postoperative decrease in serum bilirubin to $<34 \mu \mathrm{mol} / \mathrm{L}(2 \mathrm{mg} / \mathrm{dL}),{ }^{14-16}$ it remains unclear how different patient- and treatment-related factors predict progression of liver fibrosis among patients who reach normal serum bilirubin levels after PEostomy. Identifying early predictors of progressive liver fibrosis would facilitate targeting intensive postoperative monitoring to children at the greatest risk for cirrhosis and its complications. To increase our understanding of the chronic liver disease developing after successful PEostomy and to improve patient followup, we analyzed predictors for progression of liver fibrosis and studied their effect on survival of the native liver among patients who cleared their jaundice after PEostomy and underwent protocol surveillance liver biopsies.

\section{Materials and Methods}

\section{Patients and study design}

Helsinki University Children's Hospital, Finland, is a tertiary referral center for pediatric hepatobiliary surgery and is responsible for treatment of BA in Finland. ${ }^{1}$ All BA patients managed in Helsinki during 1990-2016 who cleared their jaundice after PEostomy ( $n=42,65 \%$ of all BA patients) and had undergone a follow-up liver biopsy were included in the present study. The diagnosis of BA was confirmed by intraoperative cholangiography and a histopathologic assessment of the biliary remnant. For this study, COJ was defined as plasma total bilirubin $<34 \mu \mathrm{mol} / \mathrm{L}$ $(2 \mathrm{mg} / \mathrm{dL})^{15-17}$ while living with the native liver after PEostomy. Follow-up ended at the time of last follow-up liver biopsy, when survival status of the native liver was recorded. The study protocol was approved by the hospital ethical committee.

The patient records and our prospective BA database were reviewed for relevant medical and surgical history. Use of postoperative steroids, ursodeoxycholic acid, and prophylactic antibiotics were recorded. ${ }^{1,18,19}$ The cumulative dose of steroids received postoperatively was calculated and expressed as equivalent dose of prednisolone $(\mathrm{mg} / \mathrm{kg}){ }^{20}$ Postoperative febrile episodes without any other identified cause treated with antibiotics were assumed to be cholangitis and defined as such, and their occurrence during follow-up was registered.

\section{Liver biopsies}

Patients underwent liver biopsies at PEostomy and during follow-up at the age of $1,5,10$, and 15 years, according to our postoperative follow-up protocol. ${ }^{1,10}$ Liver biopsies obtained at PEostomy and at last follow-up were included. Follow-up biopsies were core needle biopsies performed either percutaneously under ultrasonographic guidance under general anesthesia during endoscopic variceal surveillance ${ }^{10}$ or intraoperatively at time of LT. Biopsies containing at least 10 portal areas were considered representative and included in the analyses. The specimens were graded for degrees of fibrosis by using hematoxylin and eosin staining and Herovici staining. ${ }^{1}$ We applied the Metavir fibrosis staging, where stage 1 corresponds to fibrous portal expansion without formation of septa, stage 2 indicates portal fibrosis with occasional portal-to-portal septa, stage 3 portal includes fibrosis with numerous septa, and stage 4 represents cirrhosis. ${ }^{21}$ The specimens were rereviewed by two experienced pediatric pathologists blinded to clinical data until consensus was reached. Progression of liver fibrosis over follow-up time was defined as the difference between the Metavir stages at the time of PEostomy and at follow-up, and changes $\leq 0$ were considered as stable, nonprogressive fibrosis. Patients with cirrhosis (Metavir stage 4$)$ at PEostomy $(n=4)$ or no liver biopsy taken at PEostomy $(n=5)$ were excluded from the analyses of fibrosis progression. Blood count, liver biochemistry, and coagulation parameters were checked before liver biopsy, and patients were followed overnight in the hospital afterward. No complications of the liver biopsies were registered.

\section{Liver biochemistry and portal hypertension}

Liver function test results, including total and conjugated bilirubin, aspartate aminotransferase (AST), platelets, and gamma glutamyl transferase (GT) were recorded at PEostomy and at 3 and 6 months after PEostomy at the time of follow-up visits. Only measurements performed by our hospital laboratory were included. Aspartate aminotransferase-to-platelet ratio (APRI) was calculated according to the following formula: [(AST / upper normal limit of normal $) \times 100$ ] / platelet count $\left(10^{9} / \mathrm{L}\right) .{ }^{22}$ Portal hypertension was defined as the occurrence of esophageal or gastric varices at any time during follow-up or thrombocytopenia $\left(<150 \times 10^{9} / \mathrm{L}\right)$ in association with splenomegaly on ultrasonography. ${ }^{17}$

\section{Statistical methods}

Continuous data are expressed as median values with interquartile ranges and categorical data as frequencies. The Mann-Whitney $U$ test was used to compare continuous variables, and the Fisher exact test compared frequencies between subgroups. Variables showing statistically significant differences between cirrhotic patients and other patients were further analyzed in the prediction of follow-up cirrhosis. The receiving operating characteristic (ROC) curves and their areas under ROC (AUROC) curves with 95\% confidence intervals $(\mathrm{CI})$ were calculated, and the best cutoff values were defined according to the highest sum of sensitivity and specificity. Optimal cutoff values of these variables were then used in logistic regression models with follow-up cirrhosis and progression of fibrosis as primary outcomes. Statistically significant predictors from simple regression models were included in multiple regression; however, if correlation between covariates exceeded 0.65 , the variable with a lesser odds ratio (OR) was omitted from the multiple regression. Finally, cumulative survival rates of the native liver were analyzed with the Kaplan-Meier method by calculating univariate predictors of survival with the log-rank test. The level of significance was set at $P<.05$, and all analyses were performed with SPSS version 24 (IBM Corp, Armonk, NY).

\section{Ethics}

The study protocol was approved by the Helsinki University Children's Hospital, Finland, ethical committee (protocol number $345 / 03 / 1372008)$. Institutional approval was renewed on July 21, 2017 (§68 HUS/149/2017).

\section{Results}

Patient characteristics and their relation to liver fibrosis

Of the 42 patients clearing their jaundice after PEostomy, 1 was excluded because no follow-up liver biopsy was available. Of the remaining 41 patients, half $(n=22)$ were women, $85 \%$ had type 
Table 1

Characteristics of patients having cleared their jaundice according to the presence of cirrhosis at last follow-up*

\begin{tabular}{|c|c|c|c|c|}
\hline Variable & All patients $(n=41)$ & $\begin{array}{l}\text { Patients without cirrhosis at } \\
\text { follow-up }(n=19)\end{array}$ & $\begin{array}{l}\text { Patients with cirrhosis at follow-up } \\
(n=22)\end{array}$ & $P$ value \\
\hline Age at follow-up (years) & $5.2(1.6-10.2)$ & $4.5(1.4-10.3)$ & $5.4(2.2-10.1)$ & .774 \\
\hline Age at PEostomy (days) & $58(30-79)$ & $54(25-75)$ & $61(37-85)$ & .488 \\
\hline \multicolumn{5}{|l|}{ Type of biliary atresia, $n(\%)$} \\
\hline 1 or 2 & $6(15)$ & $3(16)$ & $3(14)$ & \\
\hline 3 & $35(85)$ & $16(84)$ & $19(86)$ & 1.000 \\
\hline Associated malformations, $n(\%)$ & $15(37)$ & $9(47)$ & $6(27)$ & .211 \\
\hline Polysplenia, $n(\%)$ & $7(17)$ & $3(16)$ & $4(18)$ & 1.000 \\
\hline $\begin{array}{l}\text { Total bilirubin at PEostomy }(\mu \mathrm{mol} / \mathrm{L}) \text {, } \\
{[\mathrm{mg} / \mathrm{dL}]^{\dagger}}\end{array}$ & $149(110-205)[8.7(6.4-12)]$ & $136(103-168)[8.0(6.0-9.8)]$ & 177 (139-215) [10.4 (8.1-12.6)] & .037 \\
\hline $\begin{array}{l}\text { Conjugated bilirubin at PEostomy } \\
(\mu \mathrm{mol} / \mathrm{L}),[\mathrm{mg} / \mathrm{dL}]^{\ddagger}\end{array}$ & $101(83-154)[5.9(4.9-9.0)]$ & $98(78-117)[5.7(4.6-6.8)]$ & $125(84-175)[7.3(4.9-10.2)]$ & .059 \\
\hline APRI at PEostomy $\S$ & $0.90(0.48-1.24)$ & $0.78(0.52-0.92)$ & $1.05(0.51-1.48)$ & .232 \\
\hline \multicolumn{5}{|c|}{ Metavir fibrosis stage at PEostomy, $n(\%)$} \\
\hline 1 & $2(5.6)$ & $0(0)$ & $2(10)$ & .987 \\
\hline 2 & $19(53)$ & $9(56)$ & $10(50)$ & \\
\hline 3 & $11(31)$ & $7(44)$ & $4(20)$ & \\
\hline 4 & $4(11)$ & $0(0)$ & $4(20)$ & \\
\hline \multicolumn{5}{|c|}{ Metavir fibrosis stage at follow-up, $n(\%)$} \\
\hline 1 & $3(7.3)$ & $3(16)$ & - & $<.001$ \\
\hline 2 & $9(22)$ & $9(47)$ & - & \\
\hline 3 & $7(17)$ & $7(37)$ & - & \\
\hline 4 & $22(53.7)$ & - & $22(100)$ & \\
\hline $\begin{array}{l}\text { Steroids administered after PEostomy, } n \\
\text { (\%) }\end{array}$ & $34(83)$ & $16(84)$ & $18(82)$ & 1.000 \\
\hline $\begin{array}{l}\text { Cumulative prednisolone dose after } \\
\text { PEostomy }(\mathrm{mg} / \mathrm{kg})\end{array}$ & $48.1(23.8-71.4)$ & $46.8(21.4-68.3)$ & $49.1(37.7-66.4)$ & .751 \\
\hline Time to clearance of jaundice (months) & $1.97(0.69-3.22)$ & $0.93(0.57-2.33)$ & $2.69(1.77-3.47)$ & .011 \\
\hline $\begin{array}{l}\text { Total bilirubin at } 3 \text { months }(\mu \mathrm{mol} / \mathrm{L}) \text {, } \\
{[\mathrm{mg} / \mathrm{dL}]}\end{array}$ & $11(6.0-20)[0.64(0.35-1.2)]$ & $6.0(3.0-12)[0.35(0.18-0.70)]$ & $18(10-27)[1.1(0.58-1.6)]$ & .001 \\
\hline $\begin{array}{l}\text { Conjugated bilirubin at } 3 \text { months } \\
(\mu \mathrm{mol} / \mathrm{L}),[\mathrm{mg} / \mathrm{dL}]\end{array}$ & $5.5(2.0-13)[0.32(0.12-0.76)]$ & $2.5(2.0-5.5)[0.15(0.12-0.32)]$ & $12(4.5-23)[0.70(0.26-1.4)]$ & $<.001$ \\
\hline APRI at 3 months ${ }^{\dagger}$ & $0.57(0.34-0.96)$ & $0.34(0.21-0.50)$ & $0.79(0.57-1.14)$ & .004 \\
\hline GT at 3 months $(\mathrm{U} / \mathrm{L})^{\ddagger}$ & $319(227-565)$ & $267(140-49)$ & $349(265-633)$ & .126 \\
\hline $\begin{array}{l}\text { Total bilirubin at } 6 \text { months }(\mu \mathrm{mol} / \mathrm{L}) \text {, } \\
{[\mathrm{mg} / \mathrm{dL}]}\end{array}$ & $8.0(4.0-24)[0.47(0.23-1.4)]$ & $4.0(3.0-7.5)[0.23(0.18-0.44)]$ & $14(8.0-28)[0.82(0.47-1.6)]$ & $<.001$ \\
\hline $\begin{array}{l}\text { Conjugated bilirubin at } 6 \text { months } \\
(\mu \mathrm{mol} / \mathrm{L}),[\mathrm{mg} / \mathrm{dL}]\end{array}$ & $3.0(2.0-11)[0.18(0.12-0.64)]$ & $2.0(-)[0.12(-)]$ & $8.0(3.0-22)[0.47(0.18-1.3)]$ & $<.001$ \\
\hline APRI at 6 months $^{\S}$ & $0.77(0.40-1.40)$ & $0.40(0.26-1.11)$ & $0.99(0.65-1.45)$ & .037 \\
\hline GT at 6 months $(\mathrm{U} / \mathrm{L})^{\dagger}$ & $232(114-370)$ & $153(101-232)$ & $300(209-461)$ & .005 \\
\hline Cholangitis episodes/year & $0.31(0.00-1.29)$ & $0.31(0.04-0.77)$ & $0.28(0.00-2.26)$ & .731 \\
\hline Cholangitis within last year, $n(\%)$ & $15(37)$ & $5(26)$ & $10(45)$ & .333 \\
\hline \multicolumn{5}{|c|}{ Total number of cholangitis episodes, $n(\%)$} \\
\hline 0 & $11(27)$ & $5(26)$ & $6(27)$ & \\
\hline $1-2$ & $12(29)$ & $8(42)$ & $4(18)$ & \\
\hline Three or more & $18(44)$ & $6(32)$ & $12(55)$ & .214 \\
\hline Listed for transplantation, $n(\%)$ & $10(24)$ & $0(0)$ & $10(45)$ & .001 \\
\hline
\end{tabular}

* Continuous data are reported as median with interquartile range.

$\dagger$ Results missing for 2 patients.

$\ddagger$ Results missing for 3 patients.

$\S$ Results missing for 4 patients.

${ }^{9}$ Histology at portoenterostomy (PEostomy) missing for 5 patients. APRI, aspartate aminotransferase to platelet ratio.

3 BA, and the majority presented with Metavir fibrosis stage 2 or 3 at time of PEostomy (Table 1). All patients had received ursodeoxycholic acid and prophylactic antibiotics after PEostomy, and steroids had been administered in $83 \%(34 / 41)$.

At last follow-up at median age of 5.2 (1.6-10.2) years, all patients were alive, and liver biopsy showed cirrhosis in 22 (53\%). The stage of fibrosis had remained unchanged $(n=5)$ or decreased $(n=7)$ during follow-up in $38 \%$ of patients (Table 2$)$. Follow-up cirrhosis and progression of fibrosis were unrelated with follow-up age, PEostomy age, anatomic type of BA, postoperative administration of steroids, the cumulative steroid dose, episodes of cholangitis, or associated anomalies (Tables 1 and 2), and the Metavir stage at PEostomy was less among patients whose fibrosis progressed during follow-up (Table 2). Altogether 23 patients (56\%) developed portal hypertension during follow-up and presented with cirrhosis $(18 / 23$ vs $4 / 18, P=.001)$ and progressive fibrosis $(14 / 16$ vs $6 / 16$, $P=.009$ ) more frequently than others. Patient age showed no cor- relation with follow-up stage of fibrosis stage or progression of fibrosis ( $P=$ not significant for both; Supplementary Fig).

\section{Prediction of follow-up cirrhosis}

Patients who presented with cirrhosis at follow-up had greater preoperative serum bilirubin levels, greater levels of total bilirubin, conjugated bilirubin, and APRI at 3 and 6 months after PEostomy, and greater levels of GT at 6 months after PEostomy compared with others (Table 1 ). They had also cleared their jaundice markedly slower after PEostomy than patients without cirrhosis (Table 1 ). The variables showing statistically significant differences between cirrhotic and noncirrhotic patients were further evaluated for their value in the prediction of cirrhosis by using AUROC analyses (Table 3). By applying the defined cutoffs in logistic regression models, the risk for follow-up cirrhosis was increased if total or conjugated bilirubin exceeded $170 / 120 \mu \mathrm{mol} / \mathrm{L}(10 / 7.0$ 
Table 2

Characteristics of patients having cleared their jaundice according to the progression of the Metavir stage*

\begin{tabular}{|c|c|c|c|}
\hline Variable & Patients with unprogressive fibrosis $(n=12)$ & Patients with progressive fibrosis $(n=20)$ & $P$ value \\
\hline Age at follow-up (years) & $4.4(1.3-8.1)$ & $4.3(1.6-8.0)$ & .924 \\
\hline Age at PEostomy (days) & $60(25-77)$ & $55(34-73)$ & .744 \\
\hline \multicolumn{4}{|l|}{ Type of biliary atresia, $n(\%)$} \\
\hline 1 or 2 & $2(17)$ & $2(10)$ & \multirow[t]{2}{*}{.620} \\
\hline 3 & $10(83)$ & $18(90)$ & \\
\hline Associated malformations, $n$ (\%) & $6(50)$ & $6(30)$ & .288 \\
\hline Polysplenia, $n(\%)$ & $1(8.3)$ & $3(15)$ & 1.000 \\
\hline Total bilirubin at PEostomy $(\mu \mathrm{mol} / \mathrm{L}),[\mathrm{mg} / \mathrm{dL}]$ & $132(102-168)[7.7(6.0-9.8)]$ & $176(111-211)[10(6.5-12)]$ & .107 \\
\hline Conjugated bilirubin at PEostomy $(\mu \mathrm{mol} / \mathrm{L}),[\mathrm{mg} / \mathrm{dL}]$ & $100(78-117)[5.9(4.6-6.8)]$ & $98(83-166)[5.7(4.9-9.7)]$ & .477 \\
\hline APRI at PEostomy ${ }^{\dagger}$ & $0.78(0.47-0.90)$ & $0.84(0.46-1.22)$ & .641 \\
\hline \multicolumn{4}{|l|}{ Metavir fibrosis stage at PEostomy, $n(\%)$} \\
\hline 1 & $0(0)$ & $2(10)$ & \multirow[t]{4}{*}{.048} \\
\hline 2 & $5(42)$ & $14(70)$ & \\
\hline 3 & $7(45)$ & $4(20)$ & \\
\hline 4 & - & - & \\
\hline \multicolumn{4}{|l|}{ Metavir fibrosis stage at follow-up, $n$ (\%) } \\
\hline 1 & $2(17)$ & 0 & \multirow[t]{4}{*}{$<.001$} \\
\hline 2 & $7(58)$ & 0 & \\
\hline 3 & $3(25)$ & $4(20)$ & \\
\hline 4 & $0(0)$ & $16(80)$ & \\
\hline Steroids administered after PEostomy, $n(\%)$ & $10(83)$ & $18(90)$ & 1.000 \\
\hline Cumulative prednisolone dose $(\mathrm{mg} / \mathrm{kg})$ after PEostomy & $45.9(21.4-60.4)$ & $51.5(43.6-73.8)$ & .216 \\
\hline Time to clearance of jaundice (months) & $0.65(0.50-1.93)$ & $2.69(1.63-3.47)$ & .009 \\
\hline Total bilirubin at 3 months $(\mu \mathrm{mol} / \mathrm{L}),[\mathrm{mg} / \mathrm{dL}]$ & $5.5(3.0-9.0)[0.32(0.18-0.53)]$ & $18(9.0-28)[1.1(0.5-1.6)]$ & .001 \\
\hline Conjugated bilirubin at 3 months $(\mu \mathrm{mol} / \mathrm{L}),[\mathrm{mg} / \mathrm{dL}]$ & $2.0(2.0-4.0)[0.12(0.12-0.23)]$ & $12(3.5-22)[0.7(0.2-1.3)]$ & .004 \\
\hline APRI at 3 months $\ddagger$ & $0.34(0.25-0.43)$ & $0.69(0.47-1.13)$ & .003 \\
\hline GT at 3 months $(\mathrm{U} / \mathrm{L})$ & $267(174-326)$ & $347(190-759)$ & .239 \\
\hline Total bilirubin at 6 months $(\mu \mathrm{mol} / \mathrm{L}),[\mathrm{mg} / \mathrm{dL}]$ & $3.5(3.0-7.0)[0.20(0.18-0.41)]$ & $14(6.0-27)[0.8(0.35-1.6)]$ & .011 \\
\hline Conjugated bilirubin at 6 months $(\mu \mathrm{mol} / \mathrm{L}),[\mathrm{mg} / \mathrm{dL}]$ & $2.0(-)[0.12(-)]$ & $8.0(2.0-22)[0.47(0.12-1.3)]$ & .021 \\
\hline APRI at 6 months ${ }^{\S}$ & $0.40(0.18-0.74)$ & $0.99(0.65-1.50)$ & .016 \\
\hline GT at 6 months $(\mathrm{U} / \mathrm{L})^{\dagger}$ & $151(113-227)$ & $290(108-412)$ & .061 \\
\hline Cholangitis episodes/year & $0.39(0.00-1.66)$ & $0.33(0.00-0.79)$ & .716 \\
\hline Cholangitis within last year, $n(\%)$ & $4(33)$ & $7(35)$ & 1.000 \\
\hline \multicolumn{4}{|l|}{ Total number of cholangitis episodes, $n$ (\%) } \\
\hline 0 & $4(33)$ & $6(30)$ & \multirow[t]{3}{*}{.535} \\
\hline $1-2$ & $5(42)$ & $5(25)$ & \\
\hline 3 or more & $3(25)$ & $9(45)$ & \\
\hline Listed for transplantation, $n(\%)$ & $0(0)$ & $7(35)$ & .029 \\
\hline
\end{tabular}

* Continuous data are reported as median with interquartile range. Unprogressive fibrosis is defined as the difference between Metavir stage at follow-up and at portoenterostomy (PEostomy) $\leq 0$. Patients with cirrhosis at PEostomy $(n=4)$ were excluded from the analysis.

$\dagger$ Results missing for 2 patients.

$\doteqdot$ Results missing for 1 patient.

$\S$ Results missing for 3 patients.APRI; aspartate aminotransferase to platelet ratio; GT gamma glutamyl transferase.

Table 3

AUROC with $95 \% \mathrm{CI}$ and best cutoff values for prediction of cirrhosis at follow-up

\begin{tabular}{|c|c|c|c|c|c|c|}
\hline Variable & AUROC (95\% CI) & $P$ value & Optimal cutoff & Sensitivity & Specificity & Reference \\
\hline Total bilirubin at PEostomy & $0.70(0.53-0.87)$ & .037 & $171 \mu \mathrm{mol} / \mathrm{L}(10 \mathrm{mg} / \mathrm{dL})$ & 0.62 & 0.83 & $<34 \mu \mathrm{mol} / \mathrm{L}(<2.0 \mathrm{mg} / \mathrm{dL})$ \\
\hline Conjugated bilirubin at PEostomy & $0.68(0.50-0.86)$ & .059 & $120 \mu \mathrm{mol} / \mathrm{L}(7.0 \mathrm{mg} / \mathrm{dL})$ & 0.55 & 0.90 & $<5 \mu \mathrm{mol} / \mathrm{L}(<0.3 \mathrm{mg} / \mathrm{dL})$ \\
\hline Time to COJ (months) & $0.73(0.57-0.89)$ & .011 & 1.9 months & 0.73 & 0.74 & - \\
\hline Total bilirubin at 3 months & $0.82(0.68-0.95)$ & .001 & $12.5 \mu \mathrm{mol} / \mathrm{L}(0.73 \mathrm{mg} / \mathrm{dL})$ & 0.64 & 0.90 & $<34 \mu \mathrm{mol} / \mathrm{L}(<2.0 \mathrm{mg} / \mathrm{dL})$ \\
\hline Conjugated bilirubin at 3 months & $0.83(0.70-0.96)$ & $<.001$ & $7.5 \mu \mathrm{mol} / \mathrm{L}(0.44 \mathrm{mg} / \mathrm{dL})$ & 0.70 & 0.90 & $<5 \mu \mathrm{mol} / \mathrm{L}(<0.3 \mathrm{mg} / \mathrm{dL})$ \\
\hline APRI at 3 months & $0.77(0.60-0.94)$ & .004 & 0.55 & 0.77 & 0.82 & - \\
\hline Total bilirubin at 6 months & $0.85(0.73-0.97)$ & $<.001$ & $7.5 \mu \mathrm{mol} / \mathrm{L}(0.44 \mathrm{mg} / \mathrm{dL})$ & 0.77 & 0.77 & $<34 \mu \mathrm{mol} / \mathrm{L}(<2.0 \mathrm{mg} / \mathrm{dL})$ \\
\hline Conjugated bilirubin at 6 months & $0.84(0.72-0.97)$ & $<.001$ & $2.5 \mu \mathrm{mol} / \mathrm{L}(0.15 \mathrm{mg} / \mathrm{dL})$ & 0.81 & 0.77 & $<5 \mu \mathrm{mol} / \mathrm{L}(<0.3 \mathrm{mg} / \mathrm{dL})$ \\
\hline GT at 6 months & $0.76(0.61-0.91)$ & 0.006 & $266 \mathrm{U} / \mathrm{L}$ & 0.64 & 0.82 & $<50 \mathrm{U} / \mathrm{L}$ \\
\hline APRI at 6 months & $0.70(0.51-0.89)$ & .037 & 0.63 & 0.86 & 0.63 & - \\
\hline
\end{tabular}

* The optimal cutoffs of statistically significant predictors were used in regression analyses. AUROC, areas under receiving operating characteristic; CI, confidence interval; AST, aspartate aminotransferase; APRI, AST to platelet ratio; GT, gamma glutamyl transferase.

$\mathrm{mg} / \mathrm{dL})$ at PE; $12.5 / 7.5 \mu \mathrm{mol} / \mathrm{L}(0.73 / 0.44 \mathrm{mg} / \mathrm{dL})$ at 3 months after PE; or $7.5 / 2.5 \mu \mathrm{mol} / \mathrm{L}(0.44 / 0.15 \mathrm{mg} / \mathrm{dL})$ at 6 months after PE (Table 4). APRI values exceeding 0.55 at 3 months, and APRI $\geq 0.63$ and $\mathrm{GT} \geq 266 \mathrm{U} / \mathrm{L}$ at 6 months also increased the risk for cirrhosis at follow-up (Table 4).

Statistically significant variables from simple regression were adjusted for in a multiple regression. In the final model, only conjugated bilirubin at 6 months remained a significant predictor, with values $\geq 2.5 \mu \mathrm{mol} / \mathrm{L}$ ( $\geq 0.15 \mathrm{mg} / \mathrm{dL}$ ) increasing the risk of cirrhosis 35 -fold (Table 4 ).

\section{Prediction of progressive fibrosis}

Reflecting the findings in patients with established follow-up cirrhosis, patients with progressive fibrosis had cleared their jaundice significantly later and had greater total and conjugated levels of bilirubin as well as APRI at 3 and 6 months after PEostomy than patients with stable, nonprogressive fibrosis (Table 2). The variables showing significant differences between the subgroups of patients with progression of fibrosis were analyzed for the prediction of progressive fibrosis, using the obtained cutoff values for prediction 
Table 4

Predictors of follow-up cirrhosis assessed with simple logistic regression and multiple regression*

\begin{tabular}{|c|c|c|c|c|c|}
\hline \multirow[t]{2}{*}{ Variable } & \multirow[t]{2}{*}{ Patients $(n)$} & \multicolumn{2}{|l|}{ Simple regression } & \multicolumn{2}{|c|}{ Multiple regression } \\
\hline & & OR $(95 \% \mathrm{CI})$ & $P$ value & OR $(95 \% \mathrm{CI})$ & $P$ value \\
\hline \multicolumn{6}{|l|}{ Total bilirubin at PEostomy ${ }^{\dagger}$} \\
\hline$<170 \mu \mathrm{mol} / \mathrm{L}(<10 \mathrm{mg} / \mathrm{dL})$ & 23 & 1.00 (Ref) & .007 & & \\
\hline$\geq 170 \mu \mathrm{mol} / \mathrm{L}(\geq 10 \mathrm{mg} / \mathrm{dL})$ & 16 & $8.13(1.78-37.17)$ & & & \\
\hline \multicolumn{6}{|l|}{ Conjugated bilirubin at PEostomy } \\
\hline$<120 \mu \mathrm{mol} / \mathrm{L}(<7.0 \mathrm{mg} / \mathrm{dL})$ & 25 & 1.00 (Ref) & .009 & 1.00 (Ref) & \\
\hline$\geq 120 \mu \mathrm{mol} / \mathrm{L}(\geq 7.0 \mathrm{mg} / \mathrm{dL})$ & 13 & $9.78(1.76-54.3)$ & & $20.1(0.74-543)$ & .075 \\
\hline \multicolumn{6}{|l|}{ Time to clearance of jaundice } \\
\hline$<1.9$ months & 22 & 1.00 (Ref) & & & \\
\hline$\geq 1.9$ months & 19 & $10.0(2.35-42.5)$ & .002 & & \\
\hline \multicolumn{6}{|l|}{ Total bilirubin at $3 \mathrm{mo}$} \\
\hline$<12.5 \mu \mathrm{mol} / \mathrm{L}(<0.73 \mathrm{mg} / \mathrm{dL})$ & 25 & 1.00 (Ref) & & & \\
\hline$\geq 12.5 \mu \mathrm{mol} / \mathrm{L}(\geq 0.73 \mathrm{mg} / \mathrm{dL})$ & 16 & $14.9(2.71-81.7)$ & .002 & & \\
\hline \multicolumn{6}{|l|}{ Conjugated bilirubin at $3 \mathrm{mo}$} \\
\hline$<7.5 \mu \mathrm{mol} / \mathrm{L}(<0.44 \mathrm{mg} / \mathrm{dL})$ & 23 & 1.00 (Ref) & & & \\
\hline$\geq 7.5 \mu \mathrm{mol} / \mathrm{L}(\geq 0.44 \mathrm{mg} / \mathrm{dL})$ & 18 & $11.4(2.49-52.5)$ & .002 & & \\
\hline \multicolumn{6}{|l|}{ APRI at $3 \mathrm{mo}^{\dagger}$} \\
\hline$<0.55$ & 19 & $1.00(\operatorname{Ref})$ & & 1.00 (Ref) & \\
\hline$\geq 0.55$ & 20 & $15.9(3.21-78.3)$ & .001 & $10.8(0.79-149)$ & .075 \\
\hline \multicolumn{6}{|l|}{ Total bilirubin at $6 \mathrm{mo}$} \\
\hline$<7.5 \mu \mathrm{mol} / \mathrm{L}(<0.44 \mathrm{mg} / \mathrm{dL})$ & 20 & $1.00(\operatorname{Ref})$ & & & \\
\hline$\geq 7.5 \mu \mathrm{mol} / \mathrm{L}(\geq 0.44 \mathrm{mg} / \mathrm{dL})$ & 21 & $12.8(2.88-56.4)$ & .001 & & \\
\hline \multicolumn{6}{|l|}{ Conjugated bilirubin at $6 \mathrm{mo}$} \\
\hline$<2.5 \mu \mathrm{mol} / \mathrm{L}(<0.15 \mathrm{mg} / \mathrm{dL})$ & V & 1.00 (Ref) & & 1.00 (Ref) & \\
\hline$\geq 2.5 \mu \mathrm{mol} / \mathrm{L}(\geq 0.15 \mathrm{mg} / \mathrm{dL})$ & 21 & $24.0(4.65-124)$ & $<.001$ & $35.1(1.74-708)$ & .020 \\
\hline \multicolumn{6}{|l|}{ APRI at $6 \mathrm{mo}^{\S}$} \\
\hline$<0.63$ & 13 & 1.00 (Ref) & & & \\
\hline$\geq 0.63$ & 24 & $10.0(2.05-48.9)$ & .004 & & \\
\hline \multicolumn{6}{|l|}{$\mathrm{GT}$ at $6 \mathrm{mo}^{\dagger}$} \\
\hline$<266 \mathrm{U} / \mathrm{L}$ & 17 & $1.00(\operatorname{Ref})$ & & 1.00 (Ref) & \\
\hline$\geq 266 \mathrm{U} / \mathrm{L}$ & 22 & $8.17(2.79-37.3)$ & .007 & $1.52(0.08-28.2)$ & .778 \\
\hline \multicolumn{6}{|c|}{$\begin{array}{l}* \text { Statistically significant variables from simple regression were adjusted for in multiple regression. Liver function } \\
\text { regression were chosen if significant both } 3 \text { and } 6 \text { months after portoenterostomy (PEostomy). Conjugated instead of to } \\
\text { model because of its greater OR in simple regression, and conjugated bilirubin at } 6 \text { months was chosen because of its gr } \\
\text { bilirubin measurements. ORs are reported with } 95 \% \text { confidence intervals (CI). } \\
\dagger \text { Results missing for } 2 \text { patients. } \\
\ddagger \text { Results missing for } 3 \text { patients. } \\
\S \text { Results missing for } 4 \text { patients. APRI, aspartate aminotransferase to platelet ratio; GT, gamma glutamyl transferase. }\end{array}$} \\
\hline
\end{tabular}

of cirrhosis. In simple regression, greater total and conjugated bilirubin and APRI both at 3 and 6 months after PEostomy and increased GT at 6 months after PEostomy increased the likelihood of progressive fibrosis during follow-up. Patients with Metavir stage 3 at PEostomy were more likely to have stable nonprogressive liver fibrosis compared with stages 1-2 (Table 5). In the multiple regression model, however, none of the variables remained a statistically significant predictor for progression of fibrosis (Table 5).

Survival of the native liver

In comparison with $41 \%$ of cirrhotic patients, no patient with the lesser Metavir stages of fibrosis had been listed for LT (Table 1). Accordingly, 35\% of patients with progressive fibrosis during follow-up had been listed for LT compared with none of the patients with stable, nonprogressive fibrosis (Table 2). Indications for LT included hepatopulmonary syndrome $(n=2)$, recurrent gastrointestinal bleeding $(n=4)$, repeated episodes of cholangitis $(n=2)$, ascites $(n=1)$, and poor quality of life related with endstage liver disease $(n=1)$.

Next, we assessed survival of the native liver according to the variables predictive of cirrhosis using optimal cutoffs obtained from AUROC analyses. A significantly improved survival of the native liver was observed among patients with total bilirubin < $12.5 \mu \mathrm{mol} / \mathrm{L}(<0.73 \mathrm{mg} / \mathrm{dL})$ and conjugated bilirubin $<7.5 \mu \mathrm{mol} / \mathrm{L}$ $(<0.44 \mathrm{mg} / \mathrm{dL})$ at 3 months after PEostomy and among those with total bilirubin $<7.5 \mu \mathrm{mol} / \mathrm{L}(<0.44 \mathrm{mg} / \mathrm{dL})$ and conjugated bilirubin $<2.5 \mu \mathrm{mol} / \mathrm{L}(<0.15 \mathrm{mg} / \mathrm{dL})$ at 6 months after PEostomy (Fig. 1). Survival of the native liver did not differ in relation to to- tal or conjugated bilirubin at time of PEostomy, postoperative APRI or GT values, stage of fibrosis at PE, or clearance of jaundice time $(P=$ not significant for all $)$

\section{Discussion}

To our best knowledge, this is the first study assessing the progression of histologic liver fibrosis in patients who reach normal bilirubin levels after PEostomy. Our results demonstrate that, even after serum bilirubin has decreased into normal reference range, postoperative bilirubin is a powerful predictor of liver fibrosis progression and thus survival of the native liver in patients with BA. Bilirubin levels close to the upper reference limit at 3 to 6 months after PEostomy were associated strongly with progressive fibrosis, follow-up cirrhosis, and decreased survival of the native liver. Postoperative APRI and GT showed weaker associations with followup histopathology; nevertheless, serum bilirubin remained the only variable related with cirrhosis in multiple regression and the only studied variable predictive of survival of the native liver. These findings demonstrate that, even after achieving COJ, the risk of progressive liver disease varies according to postoperative bilirubin levels and suggests that the intensiveness of patient monitoring after successful PEostomy may be guided by measuring bilirubin levels already at 3 and 6 months postoperatively.

As fibrosis progresses in most patients after COJ, or remains stable, or even resolves over time in others, our findings may have pathophysiologic implications. ${ }^{1,10}$ In BA and other chronic cholangiopathies, newly formed reactive cholangiocytes in ductular reactions are believed to promote progression of fibrosis, because 
M. Hukkinen, A. Kerola and J. Lohi et al./Surgery $x x x(x x x x) x x x$

Table 5

Predictors of the progression of fibrosis assessed with simple logistic regression and multiple regression*

\begin{tabular}{|c|c|c|c|c|c|}
\hline \multirow[t]{2}{*}{ Variable } & \multirow[t]{2}{*}{ Patients $(n)$} & \multicolumn{2}{|l|}{ Simple regression } & \multicolumn{2}{|c|}{ Multiple regression } \\
\hline & & OR $(95 \% \mathrm{CI})$ & $P$ value & OR $(95 \% \mathrm{CI})$ & $P$ value \\
\hline \multicolumn{6}{|l|}{ Liver fibrosis stage at PEostomy } \\
\hline $1-2$ & 21 & $5.60(1.15-27.4)$ & & $11.6(0.65-205)$ & .094 \\
\hline 3 & 11 & 1.00 (Ref) & .033 & $1.00($ Ref) & \\
\hline \multicolumn{6}{|c|}{ Time to clearance of jaundice after PEostomy } \\
\hline$<1.9$ months & 17 & 1.00 (Ref) & & & \\
\hline$\geq 1.9$ months & 15 & $9.29(1.57-54.8)$ & .014 & & \\
\hline \multicolumn{6}{|l|}{ Total bilirubin at $3 \mathrm{mo}$} \\
\hline$<12.5 \mu \mathrm{mol} / \mathrm{L}(<0.73 \mathrm{mg} / \mathrm{dL})$ & 19 & 1.00 (Ref) & & & \\
\hline$\geq 12.5 \mu \mathrm{mol} / \mathrm{L}(\geq 0.73 \mathrm{mg} / \mathrm{dL})$ & 13 & $16.5(1.18-155)$ & .014 & & \\
\hline \multicolumn{6}{|l|}{ Conjugated bilirubin at $3 \mathrm{mo}$} \\
\hline$<7.5 \mu \mathrm{mol} / \mathrm{L}(<0.44 \mathrm{mg} / \mathrm{dL})$ & 18 & 1.00 (Ref) & & $1.00(\operatorname{Ref})$ & .407 \\
\hline$\geq 7.5 \mu \mathrm{mol} / \mathrm{L}(\geq 0.44 \mathrm{mg} / \mathrm{dL})$ & 14 & $20.4(2.17-193)$ & .008 & $6.38(0.51-80.6)$ & \\
\hline \multicolumn{6}{|l|}{ APRI at $3 \mathrm{mo}^{\dagger}$} \\
\hline$<0.55$ & 16 & $1.00(\operatorname{Ref})$ & & $1.00(\operatorname{Ref})$ & .121 \\
\hline$\geq 0.55$ & 15 & $23.3(2.42-225)$ & .006 & $9.96(0.20-52.3)$ & \\
\hline \multicolumn{6}{|l|}{ Total bilirubin at $6 \mathrm{mo}$} \\
\hline$<7.5 \mu \mathrm{mol} / \mathrm{L}(<0.44 \mathrm{mg} / \mathrm{dL})$ & 17 & $1.00(\operatorname{Ref})$ & & & \\
\hline$\geq 7.5 \mu \mathrm{mol} / \mathrm{L}(\geq 0.44 \mathrm{mg} / \mathrm{dL})$ & 15 & $9.29(1.57-54.8)$ & .014 & & \\
\hline \multicolumn{6}{|l|}{ Conjugated bilirubin at $6 \mathrm{mo}$} \\
\hline$<2.5 \mu \mathrm{mol} / \mathrm{L}(<0.15 \mathrm{mg} / \mathrm{dL})$ & 16 & $1.00(\operatorname{Ref})$ & & & \\
\hline$\geq 2.5 \mu \mathrm{mol} / \mathrm{L}(\geq 0.15 \mathrm{mg} / \mathrm{dL})$ & 16 & $11.7(1.94-70.2)$ & .007 & & \\
\hline \multicolumn{6}{|l|}{ APRI at $6 \mathrm{mo}^{\ddagger}$} \\
\hline$<0.63$ & 11 & 1.00 (Ref) & & & \\
\hline$\geq 0.63$ & 18 & $8.75(1.53-50.1)$ & .015 & & \\
\hline \multicolumn{6}{|l|}{ GT at $6 \mathrm{mo}^{\S}$} \\
\hline$<266 \mathrm{U} / \mathrm{L}$ & 17 & $1.00($ Ref $)$ & & 1.00 (Ref) & .359 \\
\hline$\geq 266 \mathrm{U} / \mathrm{L}$ & 13 & $13.5(1.42-128)$ & 0.023 & $5.21(0.15-176)$ & \\
\hline
\end{tabular}

\footnotetext{
* Statistically significant variables from simple regression were adjusted for in multiple regression. Liver function tests with higher odds ratios (OR) in simple regression were chosen if significant both 3 and 6 months after portoenterostomy (PEostomy). ORs are reported with 95\% confidence intervals (CI).

$\dagger$ Results missing for 1 patient.

$\ddagger$ Results missing for 3 patients.

$\S$ Results missing for 2 patients. APRI, aspartate aminotransferase to platelet ratio; GT, gamma glutamyl transferase; PEostomy, portoenterostomy.
}

the extent of ductular reaction is associated closely with both the histologic stage of fibrosis and liver expression of mesenchymal markers and growth factors driving fibrogenesis. ${ }^{1,23-26}$ Indeed, the minority of patients who demonstrate less progressive fibrosis after successful PEostomy also express less pronounced ductular reaction than others. ${ }^{24,27}$ Retention of hydrophobic bile acids has been shown to promote ductular reaction-driven fibrosis in other congenital cholangiopathies. ${ }^{28}$ Accordingly, by reflecting efficient bile flow and hepatic clearance of bile acids, very low postoperative serum bilirubin levels may indicate less active ductular reaction and, therefore, slower fibrosis progression in BA. Although understanding the mechanisms promoting fibrosis in BA still warrants further research, bilirubin values within reference limits but above the median were associated strongly with progressive liver fibrosis and cirrhosis, suggesting histologic liver injury may only be reversed if very low bilirubin levels are achieved by PEostomy.

When BA patients are not categorized according to the success of PEostomy, a serum bilirubin cutoff of $34 \mu \mathrm{mol} / \mathrm{L}(2 \mathrm{mg} / \mathrm{dL})$ at 3 months after PEostomy effectively segregates those with prolonged survival of with their native liver. ${ }^{14,16}$ In contrast, early prognosticators of survival of the native liver after $\mathrm{COJ}$ have been incompletely characterized and appear to differ from those predicting LT need among unselected BA patients. In line with our findings, greater levels of serum bilirubin, AST, APRI, and GT at 3 to 5 months after PEostomy have been suggested to be associated with decreased survival of the native liver after COJ. ${ }^{29,30}$ In our patients who underwent protocol liver biopsies not warranted by their clinical status, postoperative bilirubin outperformed other liver function tests in the prediction of survival of the native liver, followup cirrhosis, and progression of fibrosis; whereas other patientrelated and treatment-related factors had no association with these outcomes. Very low serum bilirubin levels at 3 and 6 months after PEostomy were associated with a $100 \%$ 5-year survival of the native liver, demonstrating that instead of the commonly used cutoffs of 34 or $20 \mu \mathrm{mol} / \mathrm{L}$ ( 1.2 or $1.7 \mathrm{mg} / \mathrm{dL}$ ), only bilirubin levels approaching the lower limit of normal reflect an optimal postoperative outcome.

This study is limited by its relatively small sample size, the retrospective design, and the possibility of sampling error inherent to liver biopsies. Nevertheless, this work provides novel data on the progression of histologic liver fibrosis and its predictors among patients who continue to live with their native liver and develop chronic liver disease after a successful PEostomy. Of note, prospectively collected follow-up biopsies were part of our postoperative protocol and were not influenced by the patient's clinical status, allowing us to assess factors that predict progression of fibrosis after successful PEostomy. Although biopsies were obtained at variable ages, follow-up age was unrelated to the progression of fibrosis or the presence of cirrhosis.

In conclusion, among patients whose bilirubin normalized after PEostomy, total and conjugated bilirubin $<12.5 / 7.5$ $\mu \mathrm{mol} / \mathrm{L}(0.73 / 0.44 \mathrm{mg} / \mathrm{dL})$ at 3 months after PEostomy and $<7.5 / 2.5 \mu \mathrm{mol} / \mathrm{L}(0.44 / 0.15 \mathrm{mg} / \mathrm{dL})$ at 6 months after PEostomy predicted stable, nonprogressive fibrosis, absence of cirrhosis, and improved survival of the native liver. Postoperative APRI and GT levels were less consistently related to follow-up histopathology and showed no associations with survival of the native liver. Our results suggest an optimal postPEostomy outcome involves a rapid decrease of bilirubin close to the lower limit of normal. Further research is needed to individualize the follow-up of patients with BA according to their postoperative risk for progression of their liver disease. 

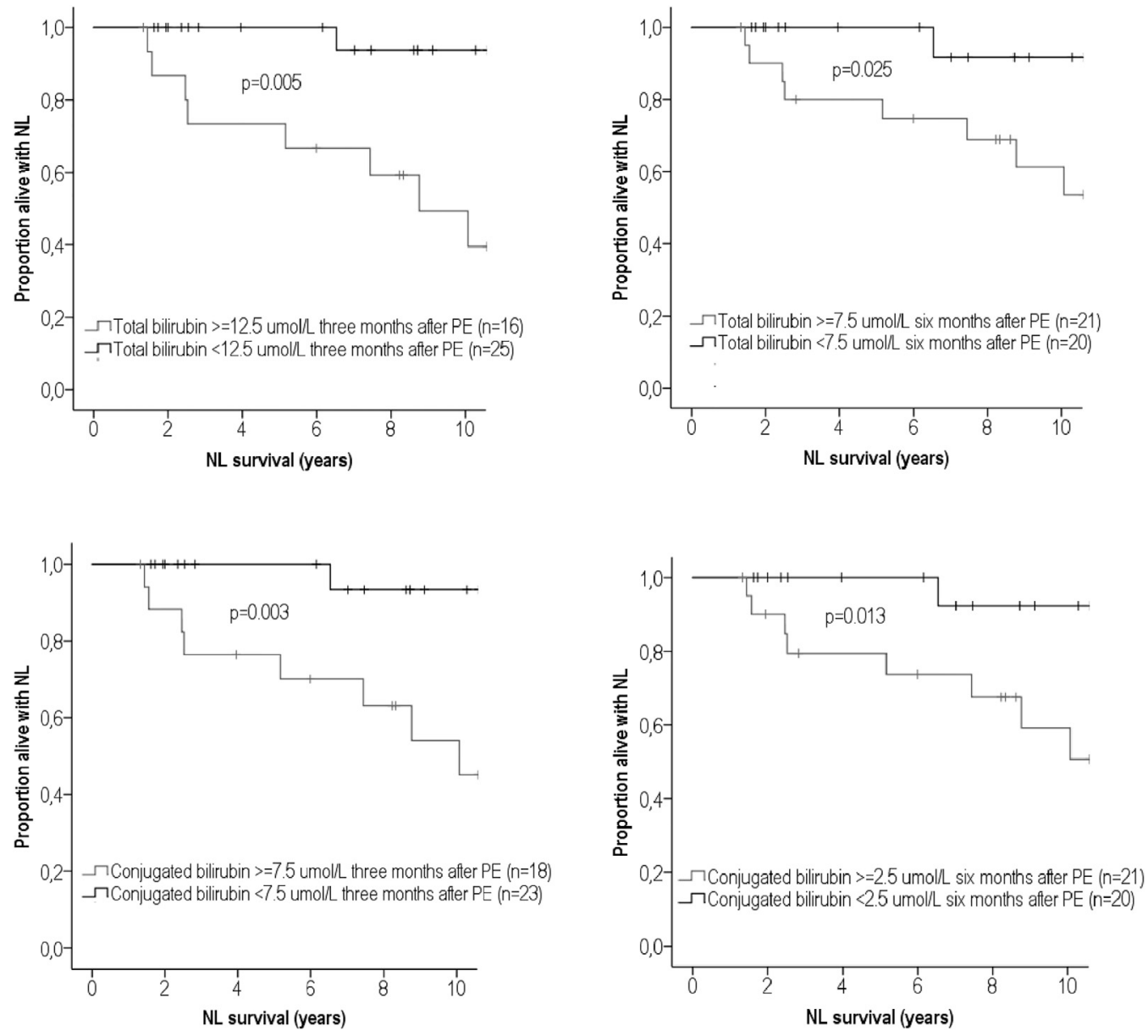

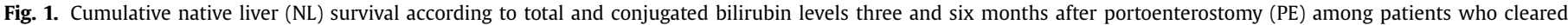

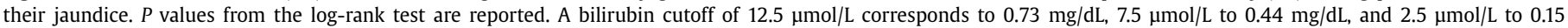
$\mathrm{mg} / \mathrm{dL}$, respectively.

\section{Supplementary materials}

Supplementary material associated with this article can be found, in the online version, at doi:10.1016/j.surg.2018.10.032.

\section{References}

1. Hukkinen M, Kerola A, Lohi J, Heikkila P, Merras-Salmio L, Jahnukainen T, et al. Treatment policy and liver histopathology predict biliary atresia outcomes: results after national centralization and protocol biopsies. J Am Coll Surg. 2017;226:46-57

2. de Vries W, de Langen ZJ, Groen H, Scheenstra R, Peeters PM, Hulscher JB, et al. Biliary atresia in the Netherlands: Outcome of patients diagnosed between 1987 and 2008. J Pediatr. 2012;160:638-644.

3. Davenport M, Ong E, Sharif K, Alizai N, McClean P, Hadzic N, et al. Biliary atresia in England and Wales: Results of centralization and new benchmark. J Pediatr Surg. 2011;46:1689-1694.

4. Chardot C, Buet C, Serinet MO, Golmard JL, Lachaux A, Roquelaure B, et al. Improving outcomes of biliary atresia: French national series 1986-2009. J Hepatol. 2013;58:1209-1217.

5. de Vries W, Homan-Van der Veen J, Hulscher JB, Hoekstra-Weebers JE, Houwen RH, Verkade HJ, et al. Twenty-year transplant-free survival rate among patients with biliary atresia. Clin Gastroenterol Hepatol. 2011;9:1086-1091.

6. Shinkai M, Ohhama Y, Take H, Kitagawa N, Kudo H, Mochizuki K, et al. Long-term outcome of children with biliary atresia who were not transplanted after the Kasai operation: >20-year experience at a children's hospital. I Pediatr Gastroenterol Nutr. 2009;48:443-450.

7. Kumagi T, Drenth JP, Guttman O, Ng V, Lilly L, Therapondos G, et al. Biliary atresia and survival into adulthood without transplantation: A collaborative multicentre clinic review. Liver Int. 2012;32:510-518.

8. Lykavieris P, Chardot C, Sokhn M, Gauthier F, Valayer J, Bernard O. Outcome in adulthood of biliary atresia: A study of 63 patients who survived for over 20 years with their native liver. Hepatology. 2005;41:366-371.

9. Ng VL, Haber BH, Magee JC, Miethke A, Murray KF, Michail S, et al. Medical status of 219 children with biliary atresia surviving long-term with their na- tive livers: Results from a North American multicenter consortium. J Pediatr. 2014; 165:539-546.

10. Lampela H, Kosola S, Heikkila P, Lohi J, Jalanko H, Pakarinen MP. Native liver histology after successful portoenterostomy in biliary atresia. J Clin Gastroenterol. 2014;48:721-728.

11. Kuo FY, Huang CC, Chen CL, Chuang JH, Riehle K, Swanson PE, et al. Immunohistochemical characterization of the regenerative compartment in biliary atresia: A comparison between Kasai procedure and transplant cases. Hum Pathol. 2015;46:1633-1639.

12. Tomita H, Masugi Y, Hoshino K, Fuchimoto Y, Fujino A, Shimojima N, et al. Long-term native liver fibrosis in biliary atresia: development of a novel scoring system using histology and standard liver tests. J Hepatol. 2014;60:1242-1248.

13. Voutilainen S, Kivisaari R, Lohi J, Jalanko H, Pakarinen MP. A prospective comparison of noninvasive methods in the assessment of liver fibrosis and esophageal varices in pediatric chronic liver diseases. J Clin Gastroenterol. 2016;50:658-663.

14. Shneider BL, Magee JC, Karpen SJ, Rand EB, Narkewicz MR, Bass LM, et al. Total serum bilirubin within 3 months of hepatoportoenterostomy predicts short-term outcomes in biliary atresia. J Pediatr. 2016;170:211-217.

15. Nightingale S, Stormon MO, O'Loughlin EV, Shun A, Thomas G, Benchimol EI, et al. Early posthepatoportoenterostomy predictors of native liver survival in biliary atresia. J Pediatr Gastroenterol Nutr. 2017;64:203-209.

16. Superina R, Magee JC, Brandt ML, Healey PJ, Tiao G, Ryckman F, et al. The anatomic pattern of biliary atresia identified at time of Kasai hepatoportoenterostomy and early postoperative clearance of jaundice are significant predictors of transplant-free survival. Ann Surg. 2011;254:577-585.

17. Shneider BL, Abel B, Haber B, Karpen SJ, Magee JC, Romero R, et al. Portal hypertension in children and young adults with biliary atresia. J Pediatr Gastroenterol Nutr. 2012;55:567-573.

18. Willot S, Uhlen S, Michaud L, Briand G, Bonnevalle M, Sfeir R, et al. Effect of ursodeoxycholic acid on liver function in children after successful surgery for biliary atresia. Pediatrics. 2008;122:e1236-e1241.

19. Bezerra JA, Spino C, Magee JC, Shneider BL, Rosenthal P, Wang KS, et al. Use of corticosteroids after hepatoportoenterostomy for bile drainage in infants with biliary atresia: The START randomized clinical trial. JAMA. 2014;311:17501759 . 
M. Hukkinen, A. Kerola and J. Lohi et al./Surgery $x x x(x x x x) x x x$

20. Miller WL, Flück CE. Adrenal cortex and its disorders. In: Sperling M, ed. Pediatric endocrinology. Amsterdam: Elsevier Inc; 2014:471-523.

21. Bedossa P, Poynard TThe METAVIR Cooperative Study Group. An algorithm for the grading of activity in chronic hepatitis C. Hepatology. 1996;24:289-293.

22. Wai CT, Greenson JK, Fontana RJ, Kalbfleisch JD, Marrero JA, Conjeevaram HS, et al. A simple noninvasive index can predict both significant fibrosis and cirrhosis in patients with chronic hepatitis C. Hepatology. 2003;38:518-526.

23. Fabris L, Spirli C, Cadamuro M, Fiorotto R, Strazzabosco M. Emerging concepts in biliary repair and fibrosis. Am J Physiol Gastrointest Liver Physiol. 2017;313:G102-G116.

24. Kerola A, Lampela H, Lohi J, Heikkilä P, Mutanen A, Jalanko H, et al. Molecular signaling of active fibrogenesis prevails in biliary atresia after a successful portoenterostomy. Surgery. 2017;162:548-556.

25. Carpino G, Cardinale V, Folseraas T, Overi D, Floreani A, Franchitto A, et al. Hepatic stem/progenitor cell activation differs between primary sclerosing and primary biliary cholangitis. Am J Pathol. 2018;188:627-639.

26. Mavila N, James D, Shivakumar P, Nguyen MV, Utley S, Mak K, et al. Expansion of prominin-1-expressing cells in association with fibrosis of biliary atresia. Hepatology. 2014;60:941-953.
27. Kerola A, Lampela H, Lohi J, Heikkila P, Mutanen A, Hagstrom J, et al. Increased MMP-7 expression in biliary epithelium and serum underpins native liver fibrosis after successful portoenterostomy in biliary atresia. J Pathol Clin Res. 2016;2:187-198.

28. Trauner M, Fuchs CD, Halilbasic E, Paumgartner G. New therapeutic concepts in bile acid transport and signaling for management of cholestasis. Hepatology. 2017;65:1393-1404.

29. Koga H, Wada M, Nakamura H, Miyano G, Okawada M, Lane GJ, et al. Factors influencing jaundice-free survival with the native liver in post-portoenterostomy biliary atresia patients: Results from a single institution. J Pediatr Surg. 2013;48:2368-2372.

30. Ihn K, Ho IG, Chang EY, Han SJ. Correlation between gamma-glutamyl transpeptidase activity and outcomes after Kasai portoenterostomy for biliary atresia. J Pediatr Surg. 2018;53:461-467. 